\title{
Prevalence of preoperative anaemia and its relation with transfusion and morbimortality in patients undergoing cardiac surgery in Hospital Clinic Barcelona.
}

\author{
De la Torre Weinreiter M, Rosales M, Chanzá Albert M, Basora M, Rovira I. \\ Department of Anesthesiology. Section of cardiovascular anaesthesia.
} Hospital Clínic de Barcelona, Spain.

1- Background: The prevalence of anaemia in the population suffering from chronic heart failure is high and, in patients undergoing cardiac surgery, it is between 16 to $54 \%^{1}$. Its relation with the evolution of the disease is well stablished and, recently, a significant number of studies suggest that anaemia may be associated with unfavourable outcomes in several clinical disorders ${ }^{1}$.

2- Goal of study: The main purpose of this study was to determine the prevalence of anaemia in patients undergoing scheduled cardiac surgery in our centre. Secondary aims were to determine the relationship between preoperative anaemia and the need for transfusion, identify any relationship between anaemia and postperative complications such as acute kidney injury (AKI) and inhospital mortality. A final aim was to establish the period of time between diagnosis of anaemia and surgery, in order to determine the therapeutic window.

3- Materials and methods: We conducted an observational, descriptive and retrospective study in Hospital Clinic Barcelona. Data was collected from the preanesthetic assessment of patients undergoing cardiac surgery between January 2017 and May 2017, after signing an informed consent accepting the use of their information for the purpose of the study.

Demographic variables were studied: age, sex, body mass index and ASA score. Further variables were obtained by blood tests: haemoglobin, hematocrit. We registered the presence of anaemia $[\mathrm{Hb}$ $<13 \mathrm{~g} / \mathrm{dL}$ ] and if it was treated. The use of blood products, AKI incidence and overall inhospital mortality were also recorded. Univariate and bivariate analysis were carried out by applying measures of central tendency (mean and median) and dispersion (standard deviation).
PREVALENCE OF ANAEMIA

Figure 1

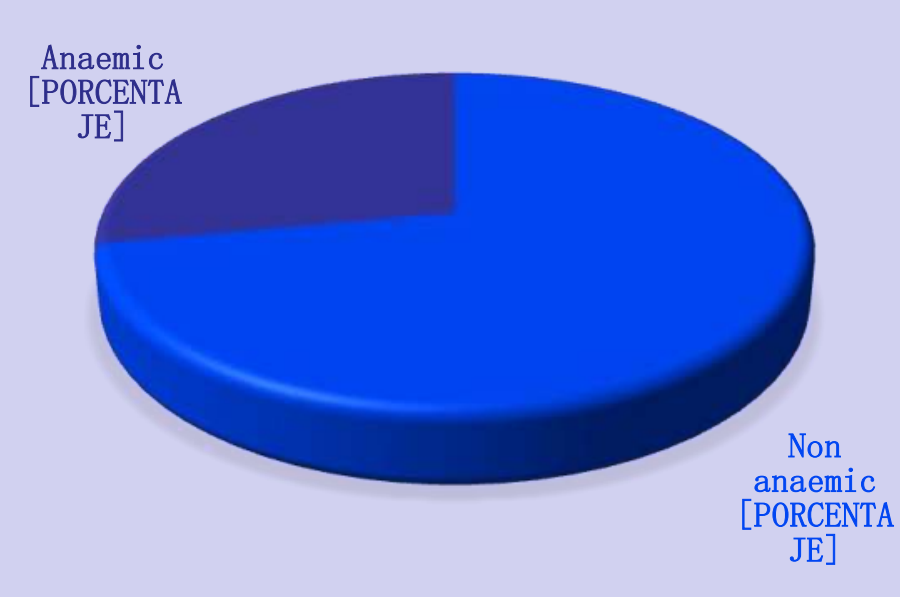

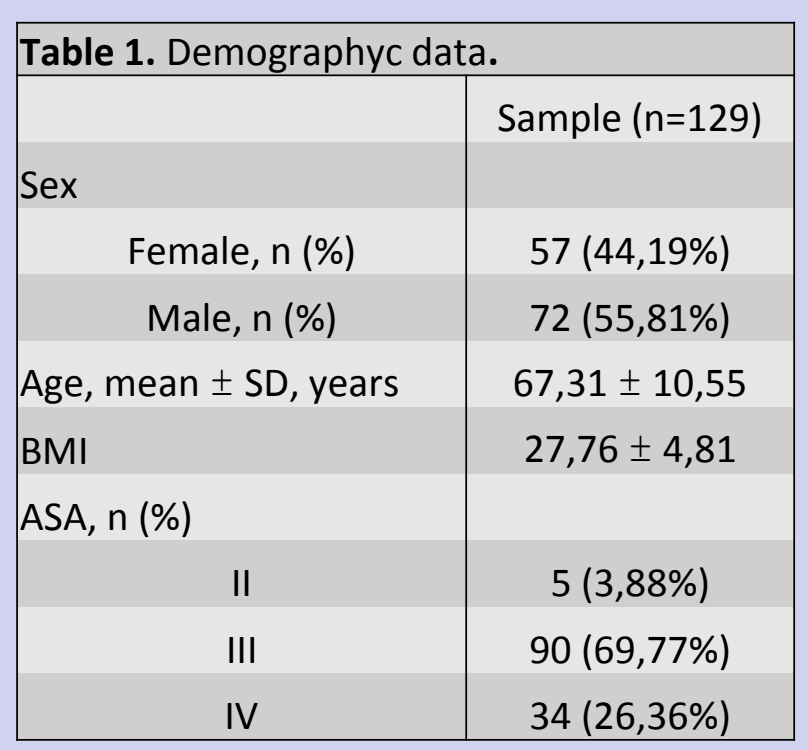

$34(26,36 \%)$
4- Results: Data was obtained from 129 patients undergoing scheduled cardiac surgery. The prevalence of anaemia obtained in the study corresponded to $27.91 \%$ [ $n=36$ ] of our population. Treatment of anaemia was started only in 7 patients [19.40\%]. Five of them with oral iron supplementation, one with erythropoietin and the last one receiving both treatments.
Mean time from diagnosis of anaemia to surgery was 23,42 days $(S D=16.61)$. The anaemic population had a higher probability of requiring blood products than non anaemic patients [OR 3.9; CI 95\% (1.73-8.77); $p<0.05]$. The presence of complications was also assessed, and it was estimated that $8.53 \%$ of all patients presented AKI. $16.66 \%$ of anaemic patients developed AKI in the perioperative period and $5.37 \%$ of non anaemic patients. The association between anaemia and incidence of AKI was analised. Anaemic patients had a higher risk of perioperative $\mathrm{AKI}$ than non anemic patients [OR=3.5; $\mathrm{Cl}$ $95 \%$ (1-12.3); $p=0.03$. There were no cases of mortality in the study.

\begin{tabular}{|l|c|c|c|}
\hline \multirow{2}{*}{ Table 2. Association between anaemia and transfusion } \\
\hline \multirow{2}{*}{ Anaemia } & \multicolumn{2}{|c|}{ Transfusion } & \multirow{2}{*}{ Total } \\
\cline { 2 - 4 } & YES & NO & 36 \\
\hline YES & 23 & 13 & 93 \\
\hline NO & 29 & 64 & 129 \\
\hline Total & 52 & 77 & 0.0006 \\
\hline Chi & & $p$ & Max \\
\hline \multirow{2}{*}{ Measure of association } & Value & \multicolumn{2}{|c|}{$95 \%$ Interval of confidence } \\
\cline { 3 - 4 } & 3.53 & Min & 8.77 \\
\hline
\end{tabular}

\begin{tabular}{|c|c|c|c|}
\hline \multirow{2}{*}{ Anaemia } & \multicolumn{2}{|c|}{ AKI } & \multirow{2}{*}{ Total } \\
\hline & YES & NO & \\
\hline YES & 6 & 30 & 36 \\
\hline NO & 5 & 88 & 93 \\
\hline Total & 11 & 118 & 129 \\
\hline $\mathrm{Chi}^{2}$ & 4.24 & $\mathrm{p}$ & 0.03 \\
\hline \multirow{2}{*}{ Measure of association } & \multirow{2}{*}{ Value } & \multicolumn{2}{|c|}{$95 \%$ Interval of confidence } \\
\hline & & Min & Max \\
\hline OR & 3.5 & 1 & 1.37 \\
\hline
\end{tabular}

5- Discussion: In this study, we found that prevalence of anemia anaemia was $27.91 \%$. The association between these variables has an OR of 3.9 , which it suggests that patients with anaemia are transfused up to almost 4 times more than those who do not. We also observed that of the group of patients with anemia, $16.66 \%$ developed $\mathrm{AKI}$ in the perioperative period and non-anemic ones $5.37 \%$, establishing the relation that patients with anaemia have 3.5 times more risk of developing AKI than a patient without anemia.

6- Conclusions: The prevalence of preoperative anemia continues to be high in patients scheduled for elective cardiac surgery. Average time from diagnosis to surgery was 23 days; It would be probably enought time to start a treatment, eventhough it was started only in $19.40 \%$ of patients. In this study, anemia did not increase mortality, but it was associated with a significant increase in transfusion of blood components and complications such as AKI. More studies are neccessary to assess the impact of anaemia in this population and develop better strategies for its treatment.

\section{Euroanaesthesia 2018 :}

Reference: 1.- Yanes G. Anemia en pacientes candidatos a cirugía cardíaca. Rev Esp Anestesiol Reanim. 2015;62(Supl 1):69-75. 